\title{
TOPOLOGICAL INVARIANT MEANS ON LOCALLY COMPACT GROUPS AND FIXED POINTS
}

\author{
JAMES C. S. WONG ${ }^{1}$
}

\begin{abstract}
A locally compact group $G$ is said to have the fixed point property if whenever $G$ acts affinely on a compact convex subset $S$ of a separated locally convex space $E$ with the map $G \times S \rightarrow S$ jointly continuous, there is a fixed point for the action. $N$. Rickert has proved that $G$ has this fixed point property if $G$ is amenable. In this paper, we study the fixed point property for actions of the algebras $L_{1}(G)$ and $M(G)$ and prove some fixed point theorems for locally compact groups.
\end{abstract}

1. Introduction. Let $G$ be a locally compact group, $G$ is said to have the fixed point property if whenever $G$ acts affinely on a compact convex subset $S$ in a separated locally convex space $E$ with the map $G \times S \rightarrow S$ (denoted by $\left.(x, s) \rightarrow T_{x}(s)\right)$ jointly or separately continuous, ${ }^{2}$ there is a point $s_{0} \in S$ such that $T_{x}\left(s_{0}\right)=s_{0}$ for any $x \in G$. N. Rickert [16] has proved that $G$ is amenable iff $G$ has the above fixed point property.

In this paper, we consider (analogous) fixed point properties for actions of the group algebra $L_{1}(G)$ and the measure algebra $M(G)$ (of all bounded regular Borel measures on $G$ ). It turns out that they are both characterisations of amenability of $G$.

For general terms in harmonic analysis, we follow Hewitt and Ross [10]. This paper is actually a sequel to the author's paper [20] and we shall freely employ the notations and definitions there.

2. Preliminaries. Let $G$ be a locally compact group, $L_{1}(G)$ its group algebra (with convolution as multiplication), $M(G)$ its measure

Received by the editors November 19, 1969.

AMS 1968 subject classifications. Primary 2220, 4635, 4680.

Key words and phrases. Locally compact groups, fixed point properties, fixed point theorems, amenability, topological left introverted spaces, convolution algebras $L_{1}(G)$ and $M(G)$, Silverman's invariant extension property.

1 Parts of the results of this paper are taken from the author's doctoral thesis at the University of British Columbia written under the direction of Professor E. E. Granirer. The author wishes to thank Professor Granirer for his valuable suggestions and encouragement during the preparation of this paper.

This research was supported in part by a National Research Council of Canada Fellowship.

${ }^{2}$ R. Ellis has proved in [5] that any such separately continuous action of $G$ is necessarily jointly continuous.

Copyright (c) 1971, American Mathematical Society 
algebra (of all bounded regular Borel measures on $G$ ), $E$ a separated locally convex space. An action of $L_{1}(G)$ on $E$ is a homomorphism $T$ of $L_{1}(G)$ into the algebra (under functional composition as multiplication) of all linear operators in $E$. Thus we have a bilinear mapping $L_{1}(G) \times E \rightarrow E$ denoted by $(\phi, s) \rightarrow T_{\phi}(s)$ such that $T_{\phi * \psi}$ $=T_{\phi} \circ T_{\psi}$ for any $\phi, \psi \in L_{1}(G)$.

Similarly we can define an action $M(G)$ on $E$. Let $J$ be any topology on $L_{1}(G)(M(G))$, we say that an action $T$ of $L_{1}(G)(M(G))$ on $E$ is Jseparately continuous if the map $L_{1}(G) \times E \rightarrow E(M(g) \times E \rightarrow E)$ is separately continuous when $L_{1}(G)(M(G))$ has the topology $\Im$.

Let $S$ be a compact convex subset of $E$, we say that $S$ is $P(G)$ invariant under the action $L_{1}(G) \times E \rightarrow E$ if $T_{\phi}(S) \subset S$ for any $\phi \in P(G)$ $=\left\{\phi \in L_{1}(G): \phi \geqq 0,\|\phi\|_{1}=1\right\}$. If $M_{0}(G)$ is the set of probability measures in $M(G)\left(\mu \in M_{0}(G)\right.$ iff $\mu \geqq 0$ and $\left.\|\mu\|=1\right)$. We say that $S$ is $M_{0}(G)$-invariant under the action $M(G) \times E \rightarrow E$ if $T_{\mu}(S) \subset S$ for any $\mu \in M_{0}(G)$. In the first case, $T$ induces an action of the convolution semigroup $P(G)$ on the compact convex subset $S$ (as affine maps now) still denoted by $T: P(G) \times S \rightarrow S$. In the second case, $T$ induces an action $T: M_{0}(G) \times S \rightarrow S$ of the convolution semigroup $M_{0}(G)$ on $S$. We shall refer to them as the induced actions.

Recall that a linear subspace $X$ of $L_{\infty}(G)$ is said to be topological left invariant if $\phi * f \in X$ for any $f \in X$ and $\phi \in L_{1}(G)$. It is said to be topological left introverted if $m_{L}(f) \in X$ for any $f \in X$ and $m \in L_{\infty}(G)^{*}$ where $m_{L}(f)$ is defined by $m_{L}(f)(g)=m\left((1 / \Delta) g^{\sim} * f\right)$. See [20] for details of these definitions.

\section{Fixed point theorems.}

THEOREM 3.1. Let $X$ be a topological left introverted and topological left invariant linear subspace of $L_{\infty}(G)$ containing the constants. Then the following conditions are equivalent:

(a) $X$ has a topological left invariant mean. ${ }^{3}$

(b) For any $\sigma\left(L_{1}, X\right)$-separately continuous action $T: L_{1}(G) \times E \rightarrow E$ of $L_{1}(G)$ on a separated locally convex space $E$ as linear operators in $E$ and any compact convex $P(G)$-invariant subset $S$ of $E$, the induced action $T: P(G) \times S \rightarrow S$ has a fixed point.

Proof. Assume that $X$ has a topological left invariant mean, then there is a net $\phi_{\alpha} \in P(G)$ such that $\phi * \phi_{\alpha}-\phi_{\alpha} \rightarrow 0$ in $\sigma\left(L_{1}, X\right)$ topology of $L_{1}(G)$ for any $\phi \in P(G) .{ }^{4}$ Consider the net $T_{\phi_{\alpha}}(s)$ where $s \in S$ is

'A mean $m$ on $X$ is called topological left invariant if $m(\phi * f)=m(f)$ for any $f \in X$, $\phi \in P(G)$.

4 For $X=L_{\infty}(G)$, this is well known. See Greenleaf [9, \$2.4]. For general $X$, this can be proved similarly. 
arbitrary but fixed. By compactness of $S$, we can assume $T_{\phi_{\alpha}}(s) \rightarrow s_{0}$ in $S$, passing to a subset if necessary. We claim that $s_{0}$ is the required fixed point of $T: P(G) \times S \rightarrow S$. For if $\phi \in P(G)$

$$
\begin{aligned}
T_{\phi}\left(s_{0}\right) & =T_{\phi}\left(\lim _{\alpha} T_{\phi_{\alpha}}(s)\right)=\lim _{\alpha} T_{\phi}\left(T_{\phi_{\alpha}}(s)\right)=\lim _{\alpha} T_{\phi * \phi_{\alpha}}(s) \\
& =\lim _{\alpha}\left\{T_{\phi * \phi_{\alpha}-\phi_{\alpha}}(s)+T_{\phi_{\alpha}}(s)\right\}=\lim _{\boldsymbol{\alpha}} T_{\phi_{\alpha}}(s)=s_{0},
\end{aligned}
$$

by $\sigma\left(L_{1}, X\right)$-separate continuity of $T: L_{1}(G) \times E \rightarrow E$, linearity of $\phi \rightarrow T_{\phi}(s)$ and the fact that $\phi * \phi_{\alpha}-\phi_{\alpha} \rightarrow 0$ in $\sigma\left(L_{1}, X\right)$ topology of $L_{1}(G)$. Hence (a) implies (b).

Conversely, let $E=X^{*}$ with weak* topology and define $T: L_{1}(G)$ $\times E \rightarrow E$ by $T_{\phi}(\mu)=l_{\phi}^{*} \mu$ (where $l_{\phi}$ is defined by $l_{\phi} f=(1 / \Delta) \phi^{\sim} * f, f \in X$, as in [20]). Since $l_{\phi * \psi}=l_{\psi} \circ l_{\phi}$ it is clear that $T$ defines an action of $L_{1}(G)$ on $E$ (linearity in $\phi$ or $\mu$ is obvious). We claim that $T$ is $\sigma\left(L_{1}, X\right)$ separately continuous. Suppose $\phi_{\alpha} \rightarrow \phi$ in $\sigma\left(L_{1}, X\right)$, then for fixed $\mu \in X^{*}$ and $f \in X$, we have

$$
T_{\phi_{\alpha}}(\mu)(f)-T_{\phi}(\mu)(f)=\mu\left(l_{\phi_{\alpha}} f\right)-\mu\left(l_{\phi} f\right)=\mu_{L}(f)\left(\phi_{\alpha}-\phi\right) \rightarrow 0
$$

since $X$ is topological left introverted. Therefore $T_{\phi_{\alpha}}(\mu) \rightarrow T_{\phi}(\mu)$ in $E$. Evidently the map $\mu \rightarrow T_{\phi}(\mu)$ is continuous for fixed $\phi$ since $l_{\phi}^{*}$ is $\omega^{*}-\omega^{*}$ continuous. Now take $S$ to be the set of all means in $X^{*}$. Then $S$ is $\omega^{*}$ compact convex and is $P(G)$-invariant under the $\sigma\left(L_{1}, X\right)$-separately continuous action $T: L_{1}(G) \times E \rightarrow E$ defined above. Consequently, if we assume (b), the induced action $T: P(G) \times S \rightarrow S$ must have a fixed point which is a topological left invariant mean on $X$. Therefore (b) implies (a).

REMARK 3.2. (i) The assumption that $X$ is topological left introverted is not needed in proving (a) implies (b).

(ii) In the proof of the theorem, we have never used the linearity of the map $T: L_{1}(G) \times E \rightarrow E$ where $(\phi, s) \rightarrow T_{\phi}(s)$, in the variable $s$ (for fixed $\phi$ ). This is not surprising since the linearity of the same map in $\phi$ (for fixed $s$ ) and the condition $T_{\phi * \psi}=T_{\phi} \circ T_{\psi}\left(\phi, \psi \in L_{1}(G)\right.$ ) together imply that $T$ is linear on the subspace $E_{1}=\left\{T_{\phi}(s): \phi \in L_{1}(G)\right\}-$ of $E$ (for each fixed $s$ ). This follows from the continuity of $T$ and the fact that

$$
\begin{aligned}
T_{\phi}\left(\alpha_{1} T_{\phi_{1}}(s)\right. & \left.+\alpha_{2} T_{\phi_{2}}(s)\right)=T_{\phi}\left(T_{\alpha_{1} \phi_{1}+\alpha_{2} \phi_{2}}(s)\right)=T_{\phi *\left(\alpha_{1} \phi_{1}+\alpha_{2} \phi_{2}\right.}(s) \\
& =T_{\alpha_{1} \phi * \phi_{1}+\alpha_{2} \phi * \phi_{2}}(s)=\alpha_{1} T_{\phi * \phi_{1}}(s)+\alpha_{2} T_{\phi * \phi_{2}}(s) \\
& =\alpha_{1} T_{\phi}\left(T_{\phi_{1}}(s)\right)+\alpha_{2} T_{\phi}\left(T_{\phi_{2}}(s)\right)
\end{aligned}
$$

for any $\alpha_{1}, \alpha_{2}$ real and $\phi_{1}, \phi_{2} \in L_{1}(G)$. The space $E_{1}$ is called the orbit of $s$. 
(iii) Notice that if the action $T: L_{1}(G) \times E \rightarrow E$ is $\sigma\left(L_{1}, L_{\infty}\right)$ separately continuous, then a fortiori it is separately continuous (i.e. when $L_{1}(G)$ has the norm topology). The converse might not be true. However, one can eliminate this apparent setback by considering the weak topology in $E$. Thus if the action $T: L_{1}(G) \times E \rightarrow E$ is separately continuous, then it is also $\sigma\left(L_{1}, L_{\infty}\right)$-separately continuous when $E$ has the topology $\sigma\left(E, E^{*}\right)[18$, Proposition 13, p. 39].

Consequently fixed point property with respect to $\sigma\left(L_{1}, L_{\infty}\right)$ separately continuous action and fixed point property with respect to separately continuous action are equivalent (compact subsets of $E$ remain compact in $\sigma\left(E, E^{*}\right)$ topology).

(iv) Theorem 3.1 is "more general" than Rickert's fixed point theorem [17, Theorem 4.2, p. 227] in the sense that it applies to any topological left introverted subspace $X$ of $L_{\infty}(G)$, for example $X$ $=L_{\infty}(G)$ itself or $X=U C B_{r}(G)$ the space of all bounded right uniformly continuous functions on $G$, while Rickert's theorem concerns only $U C B_{r}(G)$.

We naturally expect Theorem 3.1 to have an analogue for actions of $M(G)$. For general $X$, there is the difficulty of finding an analogous topology for $M(G)$ corresponding to the topology $\sigma\left(L_{1}, X\right)$ of $L_{1}(G)$. However, when $X=L_{\infty}(G)$, we have the following theorem.

TheOREM 3.3. Let $G$ be a locally compact group, then the following conditions on $G$ are equivalent:

(a) $G$ is amenable (i.e. $L_{\infty}(G)$ has a topological left invariant mean).

(b) For any separately continuous action ${ }^{5} T: L_{1}(G) \times E \rightarrow E$ of $L_{1}(G)$ on a separated locally convex space $E$ as linear operators in $E$ and any compact convex $P(G)$-invariant subset $S$ of $E$, the induced action $T: P(G) S \rightarrow S$ has a fixed point.

(c) For any separately continuous action $T: M(G) \times E \rightarrow E$ of $M(G)$ on a separated locally convex space $E$ as linear operators in $E$ and any compact convex $M_{0}(G)$-invariant subset $S$ of $E$, the induced action $T: M_{0}(G) \times S \rightarrow S$ has a fixed point.

Proof. In view of Theorem 3.2 (when $X=L_{\infty}(G)$ ) and Remark 3.3 (iii), it is clear that (a) implies (b).

Next assume (b) and let $T: M(G) \times E \rightarrow E$ be any separately continuous action of $M(G)$ on $E$. Since $L_{1}(G)$ is a subalgebra of $M(G)$, the restriction $T: L_{1}(G) \times E \rightarrow E$ is a separately continuous action of $L_{1}(G)$ on $E$. If $S$ is a compact convex $M_{0}(G)$-invariant subset of $E$,

If $\Im$ is the norm topology of $L_{1}(G)$ or $M(G)$, we use the term separately continuous action for $J$-separately continuous action. 
then $S$ is clearly $P(G)$-invariant since $P(G) \subset M_{0}(G)$. Therefore the induced action $T: P(G) \times S \rightarrow S$ must have a fixed point (by assumption (b)) which is necessarily a fixed point of $T: M_{0}(G) \times S \rightarrow S$ since $P(G)$ is an ideal of $M_{0}(G)$ (as convolution semigroup). Hence (b) implies (c).

Finally assume (c) and we want to prove (a). For each $\mu \in M(G)$, define a map $l_{\mu}: L_{\infty}(G) \rightarrow L_{\infty}(G)$ by $l_{\mu}(f)=\mu^{\sim} * f^{6}$ (see [10, Theorem 20.23]) $l_{\mu}$ is bounded linear [10, Theorem 20.12] and $l_{\mu \nu \nu}=l_{\nu} \circ l_{\mu}$ (a consequence of Fubini's Theorem). Let $E=L_{\infty}(G)^{*}$ with weak* topology. Define $T: M(G) \times E \rightarrow E$ by $T_{\mu}(m)=l_{\mu}^{*} m, \mu \in M(G), m$ $\in L_{\infty}(G)^{*}$. Then clearly $T$ is a separately continuous action of $M(G)$ on $E$. Let $S$ be the set of all means on $L_{\infty}(G)$. Then $S$ is weak* compact convex and $M_{0}(G)$-invariant under $T$. Therefore the induced action $T: M_{0}(G) \times E \rightarrow E$ has a fixed point which is evidently a topological left invariant mean on $L_{\infty}(G)$. This completes the proof.

\section{Silverman's invariant extension property.}

Definition 4.1. Let $G$ be a locally, compact group, $L_{1}(G)$ its group algebra, $M$ an abstract $M$-space with unit $e$ (see [10] for definition). A right action of $L_{1}(G)$ on $M$ is an antihomomorphism of $L_{1}(G)$ into the algebra of linear operators in $M$, denoted by $T: L_{1}(G) \times M \rightarrow M$ where $(\phi, s) \rightarrow T_{\phi}(s)$ (this means that $(\phi, s) \rightarrow T_{\phi}(s)$ is bilinear and $T_{\phi * \psi}=T_{\psi} \circ T_{\phi}$ for any $\left.\phi, \psi \in L_{1}(G)\right)$ such that $T_{\phi}: M \rightarrow M$ is a positive linear operator and $T_{\phi}(e)=e$ for any $\phi \in P(G)$. Suppose $J$ is a topology on $L_{1}(G)$. The right action $T$ is called J-weakly separately continuous when $L_{1}(G)$ has the topology $J$ and $M$ has the weak topology $\sigma\left(M, M^{*}\right)$ (see also Namioka [10] for his definition of right actions which is slightly different from ours).

If $A$ is a linear subspace of $M$ with $e \in A$, we say that $v \in A^{*}$ is a mean on $A$ if $\|v\|=v(e)=1$. $A$ is called $P(G)$-invariant under the right action $T$ if $T_{\phi}(A) \subset A$ for any $\phi \in P(G)$.

Given a J-weakly separately continuous right action $T: L_{1}(G)$ $\times M \rightarrow M$, consider the separated locally convex space $E=M^{*}$ with weak* topology $\sigma\left(M^{*}, M\right)$. It is easily verified that the right action $T$ induces an action $T^{*}: L_{1}(G) \times E \rightarrow E$ (in the sense of $\S 3$ ) with $(\phi, \mu) \rightarrow T_{\phi}^{*} \mu$ for any $\phi \in L_{1}(G), \mu \in M^{*}$. Moreover the induced action is J-separately continuous (note that since each $T_{\phi}: M \rightarrow M$ is weakweak continuous, it is continuous and hence $T_{\phi}^{*}: M \rightarrow M^{*}$ is well defined).

Theorem 4.2. Let $X$ be a topological left introverted and topological left invariant linear subspace of $L_{\infty}(G)$ containing the constants. Sup-

${ }^{6} \mu$ is defined by $\tilde{\mu}(E)=\mu\left(E^{-1}\right)$ for any Borel set $E$ in $G$. 
pose $X$ has a topological left invariant mean. Let $T: L_{1}(G) \times M \rightarrow M$ be a $\sigma\left(L_{1}, X\right)$-weakly separately continuous right action of $L_{1}(G)$ on an abstract $M$-space $M$ with unit $e$ and $A$ any $P(G)$-invariant subspace containing $e$. If $\nu \in A *$ is a mean on $A$ such that $\nu\left(T_{\phi}(s)\right)=\nu(s)$ for any $\phi \in P(G), s \in A$, then there is a mean $\mu \in M^{*}$ extending $\nu$ such that $\mu\left(T_{\phi}(s)\right)=\mu(s)$ for any $\phi \in P(G)$ and $s \in M$.

Conversely, if we assume, in addition, that $X$ is an abstract $M$ space (e.g. $X=L_{\infty}(G)$ ), then $X$ admits a topological left invariant mean if $X$ has the above "invariant extension property."

Proof. Consider the induced action $L_{1}(G) \times M^{*} \rightarrow M^{*}$ which sends $(\phi, \mu)$ to $T_{\phi}^{*} \mu$. As remarked before, this action is $\sigma\left(L_{1}, X\right)$-separately continuous when $M^{*}$ has the weak ${ }^{*}$ topology. Let $S=\left\{\mu \in M^{*}: \mu\right.$ is a mean on $M$ and $\mu$ extends $\nu\} . S \neq \varnothing$ by the Hahn-Banach Theorem. In fact $S$ is a weak ${ }^{*}$ closed convex subset of the unit ball in $M^{*}$ and is therefore weak* compact. We claim that $T_{\phi}(S) \subset S$ for any $\phi \in P(G)$. Let $\mu \in S, \phi \in P(G)$. Since $T_{\phi}: M \rightarrow M$ is positive linear and $T_{\phi}(e)$ $=e$, we have $\left\|T_{\phi}(s)\right\| \leqq\|s\|$ for any $s \in M$. Therefore $\left\|T_{\phi}\right\| \leqq 1$ and $\left\|T_{\phi}^{*} \mu\right\| \leqq\left\|T_{\phi}\right\| \cdot\|\mu\| \leqq\|\mu\|=1$. Consequently $\left\|T_{\phi}^{*} \mu\right\|=T_{\phi}^{*} \mu(e)=1$ or $T_{\phi}^{*} \mu$ is a mean on $M$. Also $T_{\phi}^{*} \mu$ extends $\nu$ since if $s \in A$, then $T_{\phi}^{*} \mu(s)$ $=\mu\left(T_{\phi}(s)\right)=\nu\left(T_{\phi}(s)\right)=\nu(s)$ (recall that $\left.T_{\phi}(A) \subset A\right)$. Therefore $S$ is $P(G)$-invariant under the action $T^{*}: L_{1}(G) \times M \rightarrow M^{*}$. By Theorem 3.2 , there is some $\mu \in S$ such that $T_{\phi}^{*} \mu=\mu$ for any $\phi \in P(G) . \mu$ is then the required extension of $\nu$.

Conversely, assume that $X$ is also an abstract $M$-space and that $X$ has this "invariant extension property," we can take $M=X$ and define a right action $L_{1}(G) \times M \rightarrow M$ by $T_{\phi}(f)=l_{\phi} f=(1 / \Delta) \phi^{\sim} * f, f \in X$ and $\phi \in L_{1}(G)$. We claim that it is $\sigma\left(L_{1}, X\right)$-weakly separately continuous. For if $\phi_{\alpha} \rightarrow \phi$ in $\sigma\left(L_{1}, X\right)$, then for any $\mu \in X^{*}, f \in X$,

$$
\mu\left(T_{\phi_{\alpha}}(f)-T_{\phi}(f)\right)=\mu\left(\frac{1}{\Delta} \tilde{\phi_{\alpha}} * f-\frac{1}{\Delta} \tilde{\phi} * f\right)=\mu_{L}(f)\left(\phi_{\alpha}-\phi\right) \rightarrow 0 .
$$

$\left(\mu_{L}(f) \in X\right.$ since $X$ is topological left introverted.) Hence $T_{\phi_{\alpha}}(f)$ $\rightarrow T_{\phi}(f)$ weakly in $M$. On the other hand, it is clear that each $T_{\phi}: M$ $\rightarrow M$ is continuous. Now choose $A$ to be the constants and define $\nu(\beta \cdot 1)=\beta$ for any $\beta \cdot 1 \in A$. Then $A$ is obviously $P(G)$-invariant under the $\sigma\left(L_{1}, X\right)$-weakly separately continuous right action $T: L_{1}(G)$ $\times M \rightarrow M$ and $\nu$ is a mean on $A$ satisfying $\nu\left(T_{\phi}(f)\right)=\nu(f)$ for any $\phi \in P(G)$ and $f \in A$. Any invariant extension $\mu$ of $\nu$ to $M=X$ is necessarily a topological left invariant mean on $X$.

REMARK 4.3. (i) The above characterisation of amenability of $G$ (i.e. when $X=L_{\infty}(G)$ ) is an analogue of a theorem of R. J. Silverman [19, Theorem 15] for amenable semigroups. 
(ii) Right actions of $M(G)$ on an abstract $M$-space with unit can also be defined and a theorem of similar type to Theorem 4.2 (with $X=L_{\infty}(G)$ ) can also be proved with obvious modifications.

\section{REFERENCES}

1. M. M. Day, Amenable semigroups, Illinois J. Math. 1 (1957), 509-544. MR 19, 1067.

2. - Fixed-point theorems for compact convex sets, Illinois J. Math. 5 (1961), 585-590. MR 25 \#1547.

3. - Correction to my paper "Fixed-point theorems for compact convex sets," Illinois J. Math. 8 (1964), 713. MR 29 \#6463.

4. N. Dunford and J. T. Schwartz, Linear operators. I: General theory, Pure and Appl. Math., vol. 7, Interscience, New York, 1958. MR $22 \# 8302$.

5. R. Ellis, Locally compact transformation groups, Duke Math J. 24 (1957), 119125. MR 19, 561.

6. E. E. Granirer, Extremely amenable semigroups, Math. Scand. 17 (1965), 177197. MR 33 \#5760.

7. — Extremely amenable semigroups. II, Math. Scand. 20 (1967), 93-113. MR 35 \#3422; MR $36 \# 1561$.

8. - Functional analytic properties of extremely amenable semigroups, Trans. Amer. Math. Soc. 137 (1969), 53-75. MR 39 \#765.

9. F. P. Greenleaf, Invariant means on topological groups and their applications, Van Nostrand, Princeton, N. J., 1969.

10. E. Hewitt and K. A. Ross, Abstract harmonic analysis. Vol. I: Structure of topological groups. Integration theory, group representations, Die Grundlehren der math. Wissenschaften, Band 115, Academic Press, New York; Springer-Verlag, Berlin, 1963. MR 28 \#158.

11. A. Hulanicki, Means and Folner condition on locally compact groups, Studia Math. 27 (1966), 87-104. MR 33 \#4178.

12. T. Mitchell, Constant functions and left invariant means on semigroups, Trans. Amer. Math. Soc. 119 (1965), 244-261. MR 33 \#1743.

13. - Fixed points and multiplicative left invariant means, Trans. Amer. Math. Soc. 122 (1966), 195-202. MR 32 \#7662.

14. - Function algebras, means, and fixed points, Trans. Amer. Math. Soc. 130 (1968), 117-126. MR 36 \#666.

15. - Topological semigroups and fixed points (to appear).

16. I. Namioka, On certain actions of semi-groups on L-spaces, Studia Math. 29 (1967), 63-77. MR 36 \#6910.

17. N. W. Rickert, Amenable groups and groups with the fixed point property, Trans. Amer. Math. Soc. 127 (1967), 221-232. MR 36 \#5260.

18. A. P. Robertson and W. J. Robertson, Topological vector spaces, Cambridge Univ. Press, New York, 1964. MR 28 \#5318.

19. R. J. Silverman, Means on semigroups and the Hahn-Banach extension property, Trans. Amer. Math. Soc. 83 (1956), 222-237. MR 18, 910.

20. James C. S. Wong, Topologically stationary locally compact groups and amenability, Trans. Amer. Math. Soc. 144 (1969), 351-363.

University of British Columbia, Vancouver, B. C., Canada

McMaster University, Hamilton, Ontario, Canada 